\title{
FORMATION FLIGHT WITH GENERATING FUNCTIONS: SOLVING THE RELATIVE BOUNDARY VALUE PROBLEM*
}

\author{
V.M. Guibout ${ }^{\dagger}$ D.J.Scheeres ${ }^{\ddagger}$
}

\begin{abstract}
The relative motion of a formation of spacecraft is studied. Using the Hamiltonian description $\mathbf{q}, \mathbf{p}, H(\mathbf{q}, \mathbf{p}, t)$ and solving for the generating functions for canonical transformations allows us to describe motion in the vicinity of a nominal trajectory as the solution to a two-point boundary value problem. We put some properties of such motion into relief before identifying an algorithm to solve this problem. The technique we are developing is suitable for use in designing and optimizing the nonlinear relative motion of a formation of spacecraft within a very general orbital setting.
\end{abstract}

\section{Introduction}

We are developing concepts that allow us to describe the relative non-linear motion of a formation of spacecraft as the solution to a two-point boundary value problem. By doing so, we can specify the initial and final positions (or velocities) and immediately know what the corresponding velocities (or positions) of an arbitrarily large collection of spacecraft must be. Having such descriptions developed analytically will allow for the efficient design and optimization of relative motion for large collections of spacecraft flying in formation. The technique we are developing is suitable for use in very general orbital settings, and essentially consists of finding a non-linear solution to the Hamilton-Jacobi partial differential equation for the generating functions of canonical transformations.

${ }^{*}$ Paper AIAA 2002-4639. Copyright (C)2002 The American Institute of Aeronautics and Astronautics Inc. All rights reserved.

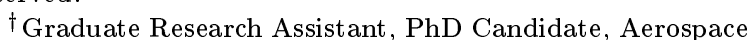
Engineering Department, The University of Michigan, guibout@umich.edu

$\ddagger$ Associate Professor, Senior member AIAA, Aerospace Engineering Department, The University of Michigan, scheeres@umich.edu
Our approach is distinct from the classical use of the generating functions as we are using them to solve the boundary value problem, whereas in previous applications they were used to solve the initial value problem instead. ${ }^{3}$

\section{Hamilton's principle and Classical Dynamics}

\section{The Hamiltonian}

A holonomic system can be described by the standard form of the Lagrange Equations:

$$
\frac{d}{d t}\left(\frac{\partial L}{\partial \dot{q}_{i}}\right)-\frac{\partial L}{\partial q_{i}}=0
$$

where the $q_{i}$ 's are generalized coordinates and $i=$ $1,2, \cdots, n$. We define the generalized momentum conjugate to $q_{i}$ as:

$$
p_{i}=\frac{\partial L}{\partial \dot{q}_{i}}
$$

Equation 1 becomes:

$$
\dot{p}_{i}=\frac{\partial L}{\partial q_{i}}
$$

Now define the Hamiltonian function as:

$$
H(q, p, t)=\sum_{i=1}^{n} p_{i} \dot{q}_{i}-L(q, \dot{q}, t)
$$

Then one can easily verify from Eq. 4 that:

$$
\left\{\begin{array}{c}
\dot{q}_{i}=\frac{\partial H}{\partial p_{i}} \\
\dot{p}_{i}=-\frac{\partial H}{\partial q_{i}}
\end{array}\right.
$$

and

$$
\frac{\partial L}{\partial t}=-\frac{\partial H}{\partial t}
$$


These $2 n$ first-order equations given in Eq. 5 are known as Hamilton's canonical equations of motion and provide a full description of the motion.

In our applications we are considering holonomic systems. Nevertheless, one can also derive a set of canonical equations of motion for a non-conservative system, where the generalized forces are not all derivable from a potential function.

\section{Canonical transformation}

Now consider transformations in phase space that preserve the Hamiltonian form of the equations of motion in the new variables. Such transformations are called canonical transformations. Let us consider a transformation from one set of coordinates $\left(q_{i}, p_{i}\right)$ to a new set $\left(Q_{i}, P_{i}\right)$ defined by equations of the form:

$$
\begin{aligned}
Q_{i} & =Q_{i}(q, p, t) \\
P_{i} & =P_{i}(q, p, t)
\end{aligned}
$$

Such transformations are often referred to as contact transformations in the literature.

Since we require the Hamiltonian form of the equations of motion in the new variables to be preserved, the following equations must be satisfied:

$$
\left\{\begin{array}{l}
\dot{Q}_{i}=\frac{\partial K}{\partial P_{i}} \\
\dot{P}_{i}=-\frac{\partial K}{\partial Q_{i}}
\end{array}\right.
$$

where $K=K(Q, P, t)$ is the Hamiltonian of the system in the new set of coordinates. To link $K$ and $H$, one must recall Hamilton's principle, ${ }^{2}$ which can be stated as:

$$
\delta I=\delta \int_{t_{0}}^{t_{1}} L d t=0
$$

where $L$ is the Lagrangian of the system. Equation 10 can be expanded using the Hamiltonian in both set of coordinates as:

$$
\begin{aligned}
& \delta \int_{t_{0}}^{t_{1}}\left(\sum_{i} p_{i} \dot{q}_{i}-H(q, p, t)\right) d t=0 \\
& \delta \int_{t_{0}}^{t_{1}}\left(\sum_{i} P_{i} \dot{Q}_{i}-K(Q, P, t)\right) d t=0
\end{aligned}
$$

This means that the integrands of the two integrals differ at most by a total time derivative of an arbitrary function $F$. Such a function is called a generating function of the transformation and must be a function of both the old and the new variables. But since the two sets of coordinates are connected by the $2 n$ transformations in Eqs. 7 and 8, $F$ depends only on $n$ old coordinates and $n$ new coordinates.

Therefore the generating functions can be written as one of the functions:

$$
F_{1}(q, Q, t), F_{2}(q, P, t), F_{3}(p, Q, t), F_{4}(p, P, t)
$$

and must satisfy a relation of the form:

$$
\sum_{i} p_{i} \dot{q}_{i}-H=\sum_{j} P_{j} \dot{Q}_{j}-K+\frac{d}{d t} F_{i}
$$

For example, expanding the total time derivative of $F_{1}$ yields:

$$
\frac{d}{d t} F_{1}(q, Q, t)=\sum_{i} \frac{\partial F_{1}}{\partial q_{i}} \dot{q}_{i}+\sum_{i} \frac{\partial F_{1}}{\partial Q_{i}} \dot{Q}_{i}+\frac{\partial F_{1}}{\partial t}
$$

We now assume the new and old coordinates $q$ and $Q$ to be independent and rewrite Eq. 14 in the following form:

$$
\begin{aligned}
p_{i} & =\frac{\partial F_{1}}{\partial q_{i}} \\
P_{i} & =-\frac{\partial F_{1}}{\partial Q_{i}} \\
K & =H+\frac{\partial F_{1}}{\partial t}
\end{aligned}
$$

If the independent variables are actually $q$ and $P$ instead of $q$ and $Q$, then the generating function is of the type $F_{2}$. Using a Legendre transformation, we find $F_{2}$ as a function of $F_{1}$ :

$$
F_{2}(q, P, t)=F_{1}(q, Q, t)+\sum_{i} P_{i} Q_{i}
$$

and then Eq. 14 becomes for $F_{2}$ :

$$
\sum_{i} p_{i} \dot{q}_{i}-H=-\sum_{j} Q_{j} \dot{P}_{j}-K+\frac{d}{d t} F_{2}
$$

which yields:

$$
\begin{aligned}
p_{i} & =\frac{\partial F_{2}}{\partial q_{i}} \\
Q_{i} & =\frac{\partial F_{2}}{\partial P_{i}} \\
K & =H+\frac{\partial F_{2}}{\partial t}
\end{aligned}
$$

The same reasoning can be applied for $F_{3}$ and $F_{4}$ if the independent variables are $(p, Q)$ or $(p, P)$ and 
we find:

$$
\begin{aligned}
q_{i} & =-\frac{\partial F_{3}}{\partial p_{i}} \\
P_{i} & =-\frac{\partial F_{3}}{\partial Q_{i}} \\
K & =H+\frac{\partial F_{3}}{\partial t} \\
q_{i} & =-\frac{\partial F_{4}}{\partial p_{i}} \\
Q_{i} & =+\frac{\partial F_{4}}{\partial P_{i}} \\
K & =H+\frac{\partial F_{4}}{\partial t}
\end{aligned}
$$

Thus, we have shown that there exist four main types of canonical transformations which allow us to switch from one coordinate set to another. Moreover, these canonical transformations must satisfy a set of equations of the form $K=H+\frac{\partial F_{i}}{\partial t}$.

\section{The Hamilton-Jacobi equation}

One can choose the new variables to be constants of motion by requiring that the new Hamiltonian $K$ be identically zero. Indeed, Hamilton's canonical equations of motion then become:

$$
\begin{aligned}
\frac{\partial K}{\partial P_{i}} & =\dot{Q}_{i}=0 \\
-\frac{\partial K}{\partial Q_{i}} & =\dot{P}_{i}=0
\end{aligned}
$$

and the new and old Hamiltonian are now related by:

$$
\frac{\partial F_{i}}{\partial t}+H(q, p, t)=0
$$

For $i=1$, we find

$$
\frac{\partial F_{1}}{\partial t}+H\left(q, \frac{\partial F_{1}}{\partial q}, t\right)=0
$$

Eq. 25 is often referred to as the Hamilton-Jacobi equation. ${ }^{2}$

One can also expand Eq. 24 for $i=2,3,4$ :

$$
\begin{aligned}
& \frac{\partial F_{2}}{\partial t}+H\left(q, \frac{\partial F_{2}}{\partial q}, t\right)=0 \\
& \frac{\partial F_{3}}{\partial t}+H\left(-\frac{\partial F_{3}}{\partial p}, p, t\right)=0 \\
& \frac{\partial F_{4}}{\partial t}+H\left(-\frac{\partial F_{4}}{\partial p}, p, t\right)=0
\end{aligned}
$$

It appears that $F_{1}\left(F_{3}\right)$ and $F_{2}\left(F_{4}\right)$ verify the same differential equation. Nevertheless, they differ in their initial conditions. From Eqns. 16 and 21 we deduce that $F_{1}$ and $F_{4}$ are undefined at time $t=0$, whereas Eqns. 19 and 20 give well-defined initial conditions for $F_{2}$ and $F_{3}$. Indeed, at the initial time the generating functions must define the identity transformation. $F_{2}$ and $F_{3}$ can generate such a transformation whereas $F_{1}$ and $F_{4}$ cannot.

Given the Hamiltonian of a system, one is able to completely solve for $F_{2}$ and $F_{3}$ starting from an initial time using the Hamilton-Jacobi equation. $F_{1}$ and $F_{4}$ can only be solved if they are known at some epoch $t \neq 0$.

In our application of the Hamilton-Jacobi equation we take the initial conditions of the trajectory to be the constants of the motion, and use the HamiltonJacobi equation to develop a local solution for the motion as a function of the initial conditions.

\section{Resolution of the boundary value problem using the Hamilton-Jacobi equation}

In our study, we investigate the Hamilton-Jacobi equation in order to solve the boundary value problem: Given $n$ initial conditions and $n$ conditions at $t=t_{f}$, solve for the $n$ remaining initial conditions and the $n$ remaining conditions at $t=t_{f}$. For instance, given an initial position and a final position, what velocities should the spacecraft have at $t=t_{0}$ and at $t=t_{f}$ to effectively reach the specified position. Such a problem is called the targeting problem and corresponds to finding the $F_{1}(q, Q)$ function between time $t_{0}$ and $t_{f}$.

\section{The boundary value problem for a one-dimensional harmonic oscillator}

We will first explore a simple example, which consists of solving the boundary value problem for a one-dimensional harmonic oscillator. Given an initial momentum $P$ and final position $q$, we solve for the initial position $Q$ and final momentum $p$. For such a problem, it is clear that $F_{2}$ is the relevant generating function.

The Hamiltonian is :

$$
H(q, p)=\frac{1}{2 m} p^{2}+\frac{k}{2} q^{2}
$$


Thus, one finds $F_{2}$ as a solution of Eq. 26:

$$
\frac{\partial F_{2}}{\partial t}+\frac{1}{2 m}{\frac{\partial F_{2}}{\partial q}}^{2}+\frac{k}{2} q^{2}=0
$$

Since $\mathrm{H}$ is a second order polynomial in $q$ and $p$, it is reasonable to look for $F_{2}$ as a polynomial in $q$ and $P$ :

$$
F_{2}(q, P, t)=\sum_{i, j} a_{i, j}(t) q^{i} P^{j}
$$

Plugging Eq. 31 into Eq. 30 and solving for each coefficient of $F_{2}$ using the following initial conditions ${ }^{1}$ : $a_{11}(0)=1, a_{i, j}(0)=0$ for all $(i, j) \neq(1,1)$ yields:

$$
\begin{aligned}
F_{2}= & -\frac{1}{2} \sqrt{k m} \tan \left(\sqrt{\frac{k}{m}} t\right) q^{2}+\sec \left(\sqrt{\frac{k}{m}} t\right) q P \\
& -\frac{1}{2} \frac{1}{\sqrt{k m}} \tan \left(\sqrt{\frac{k}{m}} t\right) P^{2}
\end{aligned}
$$

Then, Eqs. 19 allow us to solve the boundary problem:

$$
\begin{aligned}
p & =\frac{\partial F_{2}}{\partial q} \\
& =-\sqrt{k m} \tan \left(\sqrt{\frac{k}{m}} t\right) q+\sec \left(\sqrt{\frac{k}{m}} t\right) P \\
Q & =\frac{\partial F_{2}}{\partial P} \\
& =\sec \left(\sqrt{\frac{k}{m}} t\right) q-\frac{1}{\sqrt{k m}} \tan \left(\sqrt{\frac{k}{m}} t\right) P
\end{aligned}
$$

To solve the boundary value problem in position (given initial and final positions, find the initial and final velocities), we use the Legendre transformation defined in Eq. 17 to get $F_{1}$ knowing $F_{2}$. First, using Eq.33 we find $P$ as a function of $(Q, q)$ :

$P=\sqrt{k m}\left(\sec \left(\sqrt{\frac{k}{m}} t\right) \cot \left(\sqrt{\frac{k}{m}} t\right) q-\cot \left(\sqrt{\frac{k}{m}} t\right) Q\right)$

then replacing $P$ in Eq. 17 yields:

$$
\begin{aligned}
& F_{1}=\frac{1}{2} \sqrt{k m} \csc \left(\sqrt{\frac{k}{m}} t\right) \\
& \left(-2 q Q+\left(q^{2}+Q^{2}\right) \cos \left(\sqrt{\frac{k}{m}} t\right)\right)
\end{aligned}
$$

We notice that $F_{1}$ is not defined at time $t=0$.

${ }^{1}$ at $t=0 F_{2}$ is the identity transformation

$$
F_{2}(q, P, 0)=\sum_{i} q_{i} P_{i}
$$

\section{The boundary value problem} for a formation of spacecraft

\section{Formulation of the problem in terms} of Hamiltonian equations

We have seen in the previous section how to solve the boundary value problem using canonical transformation theory. Let us now apply this method to a more interesting case: a collection of spacecraft.

Given a Hamiltonian system, such as a spacecraft moving under gravitational forces, and some initial conditions $\left(Q_{0}, P_{0}\right)$, we can solve the Hamilton equations using a numerical integration scheme (at least):

$$
\dot{X}_{0}=J \nabla H_{0}
$$

where $J=\left(\begin{array}{cc}0 & I \\ -I & 0\end{array}\right), X_{0}=\left(\begin{array}{c}q\left(Q_{0}, P_{0}, t\right) \\ p\left(Q_{0}, P_{0}, t\right)\end{array}\right)$,

and $\nabla H_{0}=\left(\begin{array}{c}\frac{\partial H}{\partial q} \\ \frac{\partial H}{\partial p}\end{array}\right)\left(q\left(Q_{0}, P_{0}, t\right), p\left(Q_{0}, P_{0}, t\right), t\right)$

Suppose different initial conditions $\left(Q_{1}, P_{1}\right)$ are chosen such that:

$$
\begin{aligned}
& Q_{1}=Q_{0}+\Delta Q \\
& P_{1}=P_{0}+\Delta P
\end{aligned}
$$

Then equation 36 becomes:

$$
\dot{X}_{1}=J \nabla H_{1}
$$

where $p$ and $q$ are now functions of $\left(Q_{1}, P_{1}, t\right)$. Define two parameters $\Delta q$ and $\Delta p$ such that:

$$
\begin{aligned}
& q_{1}=q_{0}+\Delta q \\
& p_{1}=p_{0}+\Delta p
\end{aligned}
$$

where

$$
\begin{array}{ll}
q_{1}=q\left(Q_{1}, P_{1}, t\right) & p_{1}=p\left(Q_{1}, P_{1}, t\right) \\
q_{0}=q\left(Q_{0}, P_{0}, t\right) & p_{0}=p\left(Q_{0}, P_{0}, t\right)
\end{array}
$$

Equation 39 becomes:

$$
\dot{X}_{0}+\dot{X}_{h}=J \nabla H_{1}
$$

where $X_{h}=\left(\begin{array}{c}\Delta q \\ \Delta p\end{array}\right)$

Using 40, we can expand the right hand side of Eq. 43 assuming $(\Delta q, \Delta p)$ small enough for convergence of the serie:

$$
\begin{aligned}
& \nabla H\left(q_{1}, p_{1}, t\right)=\nabla H\left(q_{0}, p_{0}, t\right) \\
& \quad+\left(\begin{array}{c}
\frac{\partial^{2} H}{\partial q^{2}}\left(q_{0}, p_{0}, t\right) \Delta q+\frac{\partial^{2} H}{\partial q \partial p}\left(q_{0}, p_{0}, t\right) \Delta p \\
\frac{\partial^{2} H}{\partial q \partial p}\left(q_{0}, p_{0}, t\right) \Delta q+\frac{\partial^{2} H}{\partial p^{2}}\left(q_{0}, p_{0}, t\right) \Delta p
\end{array}\right) \\
& +\cdots
\end{aligned}
$$


Replacing into Eq. 43 yields

$$
\begin{aligned}
\dot{X}_{0} & +\dot{X}_{h}=J \nabla H_{0} \\
& +J\left(\begin{array}{c}
\frac{\partial^{2} H}{\partial q^{2}}\left(q_{0}, p_{0}, t\right) \Delta q+\frac{\partial^{2} H}{\partial q \partial p}\left(q_{0}, p_{0}, t\right) \Delta p \\
\frac{\partial^{2} H}{\partial q \partial p}\left(q_{0}, p_{0}, t\right) \Delta q+\frac{\partial^{2} H}{\partial p^{2}}\left(q_{0}, p_{0}, t\right) \Delta p
\end{array}\right) \\
& +\cdots
\end{aligned}
$$

which simplifies using Equation 36 and ignoring higher order terms:

$$
\dot{X}_{h}=J\left(\begin{array}{c}
\frac{\partial^{2} H}{\partial q^{2}}\left(q_{0}, p_{0}, t\right) \Delta q+\frac{\partial^{2} H}{\partial q \partial p}\left(q_{0}, p_{0}, t\right) \Delta p \\
\frac{\partial^{2} H}{\partial q \partial p}\left(q_{0}, p_{0}, t\right) \Delta q+\frac{\partial^{2} H}{\partial p^{2}}\left(q_{0}, p_{0}, t\right) \Delta p
\end{array}\right)
$$

This is a Hamiltonian system if and only if the right hand side can be written as the partial of a function $H_{h}$ with respect to $X_{h}$. Let

$$
H_{h}=\frac{1}{2} X_{h}\left(\begin{array}{cc}
\frac{\partial^{2} H}{\partial q^{2}}\left(q_{0}, p_{0}, t\right) & \frac{\partial^{2} H}{\partial q \partial p}\left(q_{0}, p_{0}, t\right) \\
\frac{\partial^{2} H}{\partial q \partial p}\left(q_{0}, p_{0}, t\right) & \frac{\partial^{2} H}{\partial q^{2}}\left(q_{0}, p_{0}, t\right)
\end{array}\right) X_{h}
$$

We can check that:

$$
\dot{X}_{h}=J \nabla H_{h}
$$

The expansion of the right hand side of Eq. 43 at higher order leads to:

$$
H_{h}=\sum_{\substack{i, j=0 \\ i+j \geq 2}}^{n} \frac{\Delta q^{i} \Delta p^{j}}{i ! j !} \frac{\partial^{i+j} H}{\partial q^{i} \partial p^{j}}\left(q_{0}, p_{0}, t\right)
$$

We conclude that the Hamiltonian property of the system is preserved by a Taylor series expansion. We are then free to use generating functions to solve the boundary problem ${ }^{2}$.

\section{The Hamilton Jacobi equations}

Since we have complete knowledge of $H=H_{h}$ as a function of time, we can solve for the generating functions $F_{1}, F_{2}, F_{3}$ and $F_{4}$ from equations 25- 28 (as mentioned earlier, we are missing initial conditions at $t=0$ to solve for $F_{1}$ and $F_{4}$ ).

It is now reasonable to assume that $F_{i}$ is analytic. Then when solving for $F_{i}$, we can use an expansion of $F_{i}$ of the same order as the expansion of $H_{h}$

\footnotetext{
${ }^{2}$ One must be aware that we are solving the boundary problem in terms of the relative motion and not absolute motion.
}

First we note that at the first order, $F_{i}$ is equal to zero. Indeed, suppose for instance that $F_{2}=$ $a(t) \Delta q+b(t) \Delta P$, then $\Delta q=\frac{\partial F_{2}}{\partial \Delta p}=a(t)$ and $\Delta Q=b(t)$. But $(\Delta q, \Delta p, \Delta Q, \Delta P)$ are supposed to be of the first order at least. We conclude that $a(t)=b(t)=0$.

At order $2 F_{i}$ is then a quadratic form in its two variables, without any linear term, and $H_{h}$ satisfies Eq 47.

Define $H_{h}$ and $F_{2}$ using block matrices:

$$
H_{h}=\frac{1}{2} X_{h}^{T}\left(\begin{array}{ll}
H_{q q} & H_{q p} \\
H_{p q} & H_{p p}
\end{array}\right) X_{h}
$$

and

$$
F_{2}=\frac{1}{2} Y^{T}\left(\begin{array}{ll}
F_{q q} & F_{q p} \\
F_{p q} & F_{p p}
\end{array}\right) Y
$$

where $Y=\left(\begin{array}{c}\Delta q \\ \Delta P\end{array}\right)$ and the matrix defining $H_{h}$ and $F_{2}$ are both symmetric. Then, using Eqs 19:

$$
\begin{aligned}
\Delta p & =\frac{\partial F_{2}}{\partial \Delta q} \\
& =\left(\begin{array}{ll}
F_{q q} & F_{q p}
\end{array}\right) Y
\end{aligned}
$$

Substituting into Eq. 26 yields:

$$
\begin{aligned}
0 & =Y^{T}\left\{\left(\begin{array}{ll}
\dot{F}_{q q} & \dot{F}_{q p} \\
\dot{F}_{q p}^{T} & \dot{F}_{p p}
\end{array}\right)\right. \\
& \left.+\frac{1}{2}\left(\begin{array}{ll}
I & F_{q q}^{T} \\
0 & F_{q p}^{T}
\end{array}\right)\left(\begin{array}{ll}
H_{q q} & H_{q p} \\
H_{p q} & H_{p p}
\end{array}\right)\left(\begin{array}{cc}
I & 0 \\
F_{q q} & F_{q p}
\end{array}\right)\right\} Y
\end{aligned}
$$

This equation is equivalent to the following 4 matrix equations:

$$
\begin{aligned}
\dot{F}_{q q}+\frac{1}{2}\left(H_{q q}+H_{q p} F_{q q}\right. & +F_{q q} H_{p q} \\
& \left.+F_{q q} H_{p p} F_{q q}\right)=0 \\
\dot{F}_{q p}+ & \frac{1}{2}\left(H_{q p} F_{q p}+F_{q q} H_{p p} F_{q p}\right)=0 \\
\dot{F}_{p q}+ & \frac{1}{2}\left(F_{p q} H_{p q}+F_{p q} H_{p p} F_{q q}\right)=0 \\
\dot{F}_{p p}+ & \frac{1}{2}\left(F_{p q} H_{p p} F_{q p}\right)=0
\end{aligned}
$$

where we recall that $F_{p q}=F_{q p}^{T}$ These equations allow us to solve for either $F_{2}$ or $F_{1}$ at the second order. A similar set of equations can be derived for 
$F_{3}$ and $F_{4}$ :

$$
\begin{aligned}
\dot{F}_{q q}+\frac{1}{2}\left(H_{p p}-H_{p q} F_{q q}-F_{q q} H_{q p}\right. & \\
& \left.+F_{q q} H_{q q} F_{q q}\right)=0 \\
\dot{F}_{q p}+ & \frac{1}{2}\left(-H_{p q} F_{q p}+F_{q q} H_{q q} F_{q p}\right)=0 \\
\dot{F}_{p q}+ & \frac{1}{2}\left(-F_{p q} H_{q p}+F_{p q} H_{q q} F_{q q}\right)=0 \\
\dot{F}_{p p}+ & \frac{1}{2}\left(F_{p q} H_{q q} F_{q p}\right)=0
\end{aligned}
$$

Note that the first of Eqs 54 and 55 are Ricatti equations, the second and third are non-homogeneous, time varying, linear equations and are equivalent to each other, and the last are quadrature.

If we want to generalize this method to higher order, tensor notation is required. Following are the equations derived for $F_{2}$ for a system of dimension $n$.

The Taylor expansion is now written as:

$$
f(x)=f^{0}+f^{1} \cdot \vec{x}+\left(f^{2} \cdot \vec{x}\right) \cdot \vec{x}+\left(\left(f^{3} \cdot \vec{x}\right) \cdot \vec{x}\right) \cdot \vec{x}+\cdots
$$

Applying this formula to $H(\vec{x})$ and to the canonical transformation $F_{2}=F(\vec{y})$ yields:

$$
\begin{aligned}
H(\vec{x}) & =h_{i, j} x_{i} x_{j}+h_{i, j, k} x_{i} x_{j} x_{k}+\cdots \\
F(\vec{y}) & =f_{i, j} y_{i} y_{j}+f_{i, j, k} y_{i} y_{j} y_{k}+\cdots
\end{aligned}
$$

where we assume the summation convention. Let us now express $\vec{x}=(\Delta q, \Delta p)$ as a function of $\vec{y}=$ $(\Delta q, \Delta P)$.

For all $a \leq n$ and $j=n+a$

$$
\begin{aligned}
x_{a} & =y_{a} \\
x_{j} & =\frac{\partial F}{\partial y_{a}} \\
& =f_{a, k} y_{k}+f_{k, a} y_{k} \\
& +f_{a, k, l} y_{k} y_{l}+f_{k, a, l} y_{k} y_{l}+f_{k, l, a} y_{k} y_{l} \\
& +\cdots
\end{aligned}
$$

where $n$ is the dimension of the space.

We write the Hamilton-Jacobi equation:

$$
\begin{aligned}
& \frac{\partial F}{\partial t}+H=0 \\
& \begin{array}{l}
\dot{f}_{i, j} y_{i} y_{j}+\dot{f}_{i, j, k} y_{i} y_{j} y_{k} \\
\quad+h_{i, j} x_{i} x_{j}+h_{i, j, k} x_{i} x_{j} x_{k}+\cdots=0
\end{array}
\end{aligned}
$$

Replacing $\vec{x}$ by $\vec{y}$ in equation 63 using 61 , and keep- ing only terms of order less than 3 yields:

$$
\begin{aligned}
& 0= \dot{f}_{i, j} y_{i} y_{j}+\dot{f}_{i, j, k} y_{i} y_{j} y_{k} \\
&+ h_{a, b} y_{a} y_{b}+h_{a, b, c} y_{a} y_{b} y_{c} \\
&+\left(h_{a, n+b}+h_{n+b, a}\right) y_{a}\left(f_{b, k} y_{k}+f_{k, b} y_{k}\right. \\
&\left.+f_{b, k, l} y_{k} y_{l}+f_{l, b, k} y_{k} y_{l}+f_{k, l, b} y_{k} y_{l}\right) \\
&+ h_{n+a, n+b}\left(f_{a, k} y_{k}+f_{k, a} y_{k}\right. \\
&\left.+f_{a, k, l} y_{k} y_{l}+f_{l, a, k} y_{k} y_{l}+f_{k, l, a} y_{k} y_{l}\right) \\
&\left(f_{b, m} y_{m}+f_{m, b} y_{m}+f_{b, m, p} y_{m} y_{p}\right. \\
&\left.+f_{p, b, m} y_{m} y_{p}+f_{m, p, b} y_{m} y_{p}\right) \\
&+\left(h_{n+a, b, c}+h_{c, n+a, b}+h_{b, c, n+a}\right) y_{b} y_{c} \\
&\left(f_{a, k} y_{k}+f_{k, a} y_{k}\right) \\
&+\left(h_{n+a, n+b, c}+h_{n+b, c, n+a}+h_{c, n+a, n+b}\right) \\
& y_{c}\left(f_{a, k} y_{k}+f_{k, a} y_{k}\right)\left(f_{b, l} y_{l}+f_{l, b} y_{l}\right) \\
&+ h_{n+a, n+b, n+c}\left(f_{a, k} y_{k}+f_{k, a} y_{k}\right) \\
&\left(f_{b, l} y_{l}+f_{l, b} y_{l}\right)\left(f_{c, m} y_{m}+f_{m, c} y_{m}\right)
\end{aligned}
$$

We notice that the equations of order 2 are the same as the ones found previously. The equations of order 3 are given explicitly in the appendix. The process of deriving equations for $F$ can be continued to arbitrarily high order using a symbolic manipulation program (we have implemented and solved the expansion to order 8 using Mathematica).

\section{The initial value problem}

\section{The state-transition matrix}

Another approach to the study of relative motion of spacecraft is to use the State Transition Matrix. This method is developed in ${ }^{1}$ for the case of a spacecraft moving in a point mass gravity field. R. Battin derives differential equations which allow one to compute the state transition matrix $\Phi\left(t, t_{0}\right)$. Then we have:

$$
\left(\begin{array}{c}
\Delta q \\
\Delta p
\end{array}\right)=\Phi\left(\begin{array}{c}
\Delta Q \\
\Delta P
\end{array}\right)
$$

Thus, inverting some blocks of the $\Phi$ matrix allows us to solve the boundary value problem. For instance, if $\Phi=\left(\begin{array}{ll}\Phi_{r r} & \Phi_{r v} \\ \Phi_{v r} & \Phi_{v v}\end{array}\right)$, then,

$$
\begin{aligned}
\Delta p & =\Phi_{v r} \Phi_{r r}^{-1} \Delta q+\left(\Phi_{v v}-\Phi_{v r} \Phi_{r r}^{-1} \Phi_{r v}\right) \Delta P \\
\Delta Q & =\Phi_{r r}^{-1} \Delta q-\Phi_{r r}^{-1} \Phi_{r v} \Delta P
\end{aligned}
$$

This method assumes we have computed the state transition matrix $\Phi$ with sufficient accuracy to invert 
it. Moreover, it only provides an approximation of order 2 for the solution of the boundary problem.

Battin $^{1}$ derived some interesting properties of this boundary value problem, in the next section, we will prove that these properties are actually specific to all Hamiltonian systems.

\section{Some properties of the boundary value problem}

In, ${ }^{1}$ Battin shows that one can define the matrices $C$ and $\tilde{C}$ as:

$$
\begin{aligned}
\tilde{C} & =\Phi_{v r} \Phi_{r r}^{-1} \\
C & =\Phi_{v v} \Phi_{r v}^{-1}
\end{aligned}
$$

Then, given $\Delta P=0, \tilde{C} \Delta q=\Delta p$ and given $\Delta Q=0$, $C \Delta q=\Delta p$.

$\mathrm{He}$ also proves that these matrices verify the $\mathrm{Ri}$ catti equation, and are therefore symmetric.

Using our generating function approach, we show that these matrices are just coefficients of the Taylor expansion of $F_{1}, F_{2}, F_{3}$ and $F_{4}$.

From Eqs. $61^{3}$ :

$$
\begin{aligned}
\Delta p_{a} & =x_{n+a} \\
& =\frac{\partial F}{\partial y_{a}} \\
& =\left(f_{a, k}+f_{k, a}\right) y_{k} \\
& =2 f_{a, k} y_{k} \\
\Delta p & =F_{q q} \Delta q+F_{q p} \Delta P \\
\Delta Q_{a} & =\frac{\partial F}{\partial y_{n+a}} \\
& =\left(f_{n+a, k}+f_{k, n+a}\right) y_{k} \\
& =2 f_{n+a, k} y_{k} \\
\Delta Q & =F_{p q} \Delta q+F_{p p} \Delta P
\end{aligned}
$$

where we recall that

$$
F=f_{i, j} y_{i} y_{j}=\frac{1}{2} Y^{T}\left(\begin{array}{cc}
F_{q q} & F_{q p} \\
F_{p q} & F_{p p}
\end{array}\right) Y, F_{q p}^{T}=F_{p q}
$$
and $F_{q q}$ and $F_{p p}$ are symmetric.

Solving for $(\Delta q, \Delta p)$ yields:

$$
\begin{aligned}
& \Delta q=F_{q p}^{-T} \Delta Q-F_{q p}^{-T} F_{p p} \Delta P \\
& \Delta p=F_{q q} F_{q p}^{-T} \Delta Q+\left(F_{q p}-F_{q q} F_{p q}^{-T} F_{p p}\right) \Delta P
\end{aligned}
$$

We deduce the expression of $\Phi$ :

$$
\left\{\begin{array}{l}
\Phi_{r v}=-F_{q p}^{-T} F_{p p} \\
\Phi_{r r}=F_{q p}^{-T} \\
\Phi_{v v}=F_{q p}-F_{q q} F_{q p}^{-T} F_{p p} \\
\Phi_{v r}=F_{q q} F_{q p}^{-T}
\end{array}\right.
$$

${ }^{3}$ keeping only the second order terms
We conclude that

$$
\tilde{C}=\Phi_{v r} \Phi_{r r}^{-1}=F_{q q}
$$

In the same manner, but using $F_{1}$, we show that:

$$
C=\Phi_{v v} \Phi_{r v}^{-1}=F_{q q}
$$

where $F_{q q}$ is the first block of $F_{1}$.

Thus, we have shown that $C$ and $\tilde{C}$ are symmetric by nature, and moreover that they verify the Ricatti equation given in Eq. $54^{4}$

\section{Some computations}

Using the theory we have developed in the previous section, we now numerically solve the boundary value problem to order 4 . Before doing any computation, we should first note that:

1. $F_{1}$ and $F_{4}$ are undefined at $t=0$.

2. $F_{2}$ and $F_{3}$ are well-defined at $t=0$.

3. It is possible to transform from one generating function to another using the Legendre transformation in Eq. 17.

4. It can be proved that a maximal solution to the differential equations defining $F_{i}$ exists, but there is no guarantee that the solution is defined for all time.

Numerical integration of Eqs. 54 provides a solution at order 2 to the relative boundary value problem. To get higher order terms, we use higher order expansion of $H$ and $F$ in Eqns 58.

We used the following procedure to check the validity of our computations. Given a nominal trajectory, we first solve for $F_{2}$. Then, we integrate another trajectory with different initial conditions (called the perturbed trajetory). Those two integrations gived full knowledge of $(\Delta Q, \Delta P)$ and $(\Delta q, \Delta p)$ at every instant. Then, using only $(\Delta q, \Delta P)$ we solve for $(\Delta p, \Delta Q)$ using $F_{2}$. Comparing the values of $\Delta p$ and $\Delta Q$ found using both methods results in a set of plots for the error in final momentum $\Delta p$ and a set of plots for the error in initial position $\Delta Q$. The magnitude of this error represents the error in solving the boundary value problem using our approach. The following initial conditions were used :

\footnotetext{
${ }^{4}$ In the particular case of a gravitational problem, $H_{q p}=0$ and $H_{p p}=I$
} 
- for the nominal trajectory:

$$
Q_{0}=(6500 \mathrm{~km}, 0 \mathrm{~km}), P_{0}=(0, \sqrt{\mu / 6500})
$$

- for the perturbed trajectory:

$$
Q_{1}=(6550 \mathrm{~km}, 0 \mathrm{~km}), P_{1}=(0, \sqrt{\mu / 6500})
$$

where $\mu=4 \cdot 10^{5} \mathrm{~km}^{3} \mathrm{~s}^{-2}$.

The nominal and perturbed trajectories are plotted in figure 1. We note two features of our solution.

First, we experience a singularity around $1500 \mathrm{sec}$ in solving for $F_{2}$ (Fig. 3). Eq. 66 shows that the singularity of $F_{2}$ is directly related to the non invertibility of $\phi_{r r}$ that is to:

$$
\operatorname{det}\left(\Phi_{r r}\right)=0
$$

This corresponds to there being multiple solutions to the boundary value problem at this time. Figure 2 shows the determinant of the four block matrices of the matrix $\phi$ using the initial conditions stated above (In the particular case of a circular orbit, $\left.\operatorname{det}\left(\phi_{r r}\right)=\operatorname{det}\left(\phi_{v v}\right)\right)$. In a similar way, one can relate the singularities of every $F_{i}$ with the non invertibility of a block matrix within the state transition matrix. The singularities of $F_{1}$ correspond to $\operatorname{det}\left(\phi_{r v}\right)=0$, the one of $F_{3}$ correspond to $\operatorname{det}\left(\phi_{v v}\right)=0$ and the one of $F_{4}$ correspond to $\operatorname{det}\left(\phi_{v r}\right)=0$.

One way to get around this difficulty is to solve for $F_{i}$ until a singularity is approached, then evaluate the value of $F_{j}$ at a time prior to the singularity and propagate $F_{j}$ to a time where transformation back to $F_{i}$ is needed. The issue of evaluating $F_{j}$ at time $t$ with the knowledge of $F_{i}$ at the same instant reduces to a problem of inversion of series. For instance, given $F_{2}$ we find $F_{1}$ using the Legendre transformation Eq. 17 and substituting in it $\Delta P$ as a function of $(\Delta q, \Delta Q)$. To do so one must invert the following relation $\Delta Q=\frac{\partial F 2(\Delta q, \Delta P)}{\partial \Delta P}$ as a series. Such transformations are well defined and have been dealt with by V.A. Brumberg. ${ }^{5}$ This method is reliable only if the singularities of $F_{1}, F_{2}, F_{3}$ and $F_{4}$ do not occur at the same time. Figure 2 shows that there is always one block of the state transition matrix with a determinant greater than 1 .

Second, the accuracy of our solution to the boundary value problem increases dramatically as we increase the order of our solution for $F_{2}$, giving a numerical indication that our approach is converging onto the true solution over its interval of definition. Figures 4-6 represent the error in solving the boundary problem using $F_{2}$ for $t f \in(2500,3500)$. To obtain such plots, we needed to avoid the singularities discussed above. We integrate $F_{2}$ from $t=0$ to $t=100 \mathrm{sec}$, then we used a Legendre transformation to find $F_{1}$ at $t=100 \mathrm{sec}$. We integrate $F_{1}$ up to $t=2500 \mathrm{sec}$ (we recall that $F_{2}$ was singular at $t=1500 \mathrm{sec}$ ) and use again the Legendre transformation to find $F_{2}$ at that time $t=2500 \mathrm{sec}$. We finally integrate $F_{2}$ up to $3500 \mathrm{sec}$. These figures demonstrate the convergence of our algorithm across multiple singularities.

\section{Conclusion}

This paper derives a novel application of the Hamilton-Jacobi Equation to solve the problem of non-linear targeting for a formation of spacecraft. The application constructs direct solutions for the Taylor Series expansion of the generating function to arbitrarily high order in displacements from a nominal, numerically defined trajectory. By extending the procedure to high degree in powers of the specified boundary variables, we are able to immediately generate non-linear solutions to boundary value problems. By formulating the problem as a set of ordinary differential equations, we are able to generate time histories of the boundary value problem as well. In this paper we demonstrate the basic theory and provide an example that shows its convergence. We demonstrate the generation of high order solutions for the $F_{i}$ across multiple singularities. In the future we will resolve the singularities with the aid of higher order expansions. Future work will also explore the application of this approach to the initial value problem. 


\section{A The Hamilton-Jacobi equa- tions at order 3}

Define the following variables:

$$
\begin{aligned}
& A_{i, j, k}= \\
& h_{n+a, n+b, n+c}\left(f_{a, i}+f_{i, a}\right)\left(f_{b, j}+f_{j, b}\right)\left(f_{c, k}+f_{k, c}\right) \\
& B_{i, j, k}= \\
& h_{n+a, n+b}\left(f_{a, i}+f_{i, a}\right)\left(f_{b, j, k}+f_{j, k, b}+f_{k, b, j}\right) \\
& C_{i, j, k}= \\
& h_{n+a, n+b}\left(f_{b, i}+f_{i, b}\right)\left(f_{a, j, k}+f_{j, k, a}+f_{k, a, j}\right) \\
& D_{a, i, j}=\left(h_{a, n+b, n+c}+h_{n+c, a, n+b}+h_{n+b, n+c, a}\right) \\
& \quad\left(f_{b, i}+f_{i, b}\right)\left(f_{c, j}+f_{j, c}\right) \\
& E_{a, i, j}= \\
& \left(h_{a, n+b}+h_{n+b, a}\right)\left(f_{b, i, j}+f_{j, b, i}+f_{i, j, b}\right) \\
& G_{a, b, i}= \\
& \left(h_{a, b, n+c}+h_{b, n+c, a}+h_{n+c, a, b}\right)\left(f_{c, i}+f_{i, c}\right) \quad(76)
\end{aligned}
$$

Keeping only third order terms in equation 64 yields:

$$
\begin{aligned}
& \dot{f}_{i, j, k} y_{i} y_{j} y_{k}+\left(A_{i, j, k}+B_{i, j, k}+C_{i, j, k}\right) y_{i} y_{j} y_{k} \\
& +\left(D_{a, i, j}+E_{a, i, j}\right) y_{a} y_{i} y_{j}+G_{a, b, i} y_{a} y_{b} y_{i} \\
& +h_{a, b, c} y_{a} y_{b} y_{c}=0
\end{aligned}
$$

We deduce the coefficients of $y_{i} y_{j} y_{k}$ :

- Coefficients of $y_{i \leq n}^{3}$

$$
\begin{aligned}
A_{i, i, i} & +B_{i, i, i}+C_{i, i, i}+D_{i, i, i} \\
& +E_{i, i, i}+\dot{f}_{i, i, i}+G_{i, i, i}+h_{i, i, i}=0
\end{aligned}
$$

- Coefficients of $y_{i>n}^{3}$

$$
A_{i, i, i}+B_{i, i, i}+C_{i, i, i}+\dot{f}_{i, i, i}=0
$$

- Coefficients of $y_{i \leq n}^{2} y_{j \leq n}$

$$
(A+B+C+D+E+\dot{f}+G+h)_{\tau(i, i, j)}=0
$$

where $\tau(i, j, k)$ represents all the distinct permutations of $(i, j, k)$, that is $A_{\tau(i, j, k), l}=$ $A_{i, j, k, l}+A_{i, k, j, l}+A_{k, i, j, l}+A_{k, j, i, l}+A_{j, k, i, l}+$ $A_{j, i, k, l}$ but $A_{\tau(i, i, j), l}=A_{i, i, j, l}+A_{i, j, i, l}+A_{j, i, i, l}$

- Coefficients of $y_{i \leq n}^{2} y_{j>n}$

$$
\begin{aligned}
& (A+B+C+\dot{f})_{\tau(i, i, j)} \\
& +(D+E)_{i, \tau(i, j)}+G_{i, i, j}=0
\end{aligned}
$$

- Coefficients of $y_{i \leq n} y_{j \leq n} y_{k \leq n}$ :

$$
(A+B+C+D+E+\dot{f}+G+h)_{\tau(i, j, k)}=0
$$

- Coefficients of $y_{i \leq n} y_{j \leq n} y_{k>n}$

$$
\begin{aligned}
& (A+B+C+\dot{f})_{\tau(i, j, k)}+(D+E)_{i, \tau(j, k)} \\
& +(D+E)_{j, \tau(i, k)}+G_{\tau(i, j), k}=0
\end{aligned}
$$

- Coefficients of $y_{i>n}^{2} y_{j \leq n}$

$$
(A+B+C+\dot{f})_{\tau(i, i, j)}+(E+D)_{j, i, i}=0
$$

- Coefficients of $y_{i>n}^{2} y_{j>n}$

$$
(A+B+C+\dot{f})_{\tau(i, i, j)}=0
$$

- Coefficients of $y_{i \leq n} y_{j>n} y_{k>n}$

$$
(A+B+C+\dot{f})_{\tau(i, j, k)}+(D+E)_{i, \tau(j, k)}=0
$$

- Coefficients of $y_{i>n} y_{j>n} y_{k>n}$

$$
(A+B+C+\dot{f})_{\tau(i, j, k)}=0
$$

Eqs 78-87 allow to solve for $F_{2}$ or $F_{1}$.

\section{References}

[1] R. Battin. An introduction to the Mathematics and Methods of Astrodynamics. Sec 9-5, 9-6. Revised Edition, AIAA, 1999.

[2] D. Greenwood. Classical Dynamics. PrenticeHall, 1977.

[3] H. Goldstein. Classical Mechanics. AddisonWesley, 1965.

[4] R. Moulton. Differential equations. Dover Publications, 1930.

[5] V. A. Brumberg. Analytical techniques of $\mathrm{Ce}$ lestial Mechanics. Sec 1. Springer,1995. 


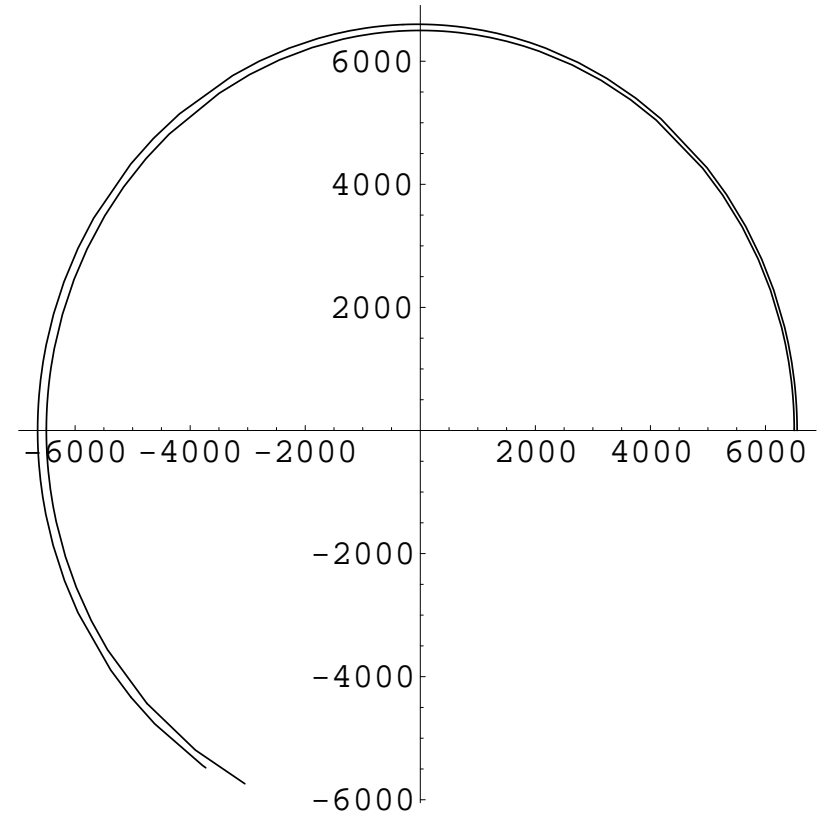

Figure 1: Nominal and perturbed trajectories, $t \in(0,3500 \mathrm{sec})$

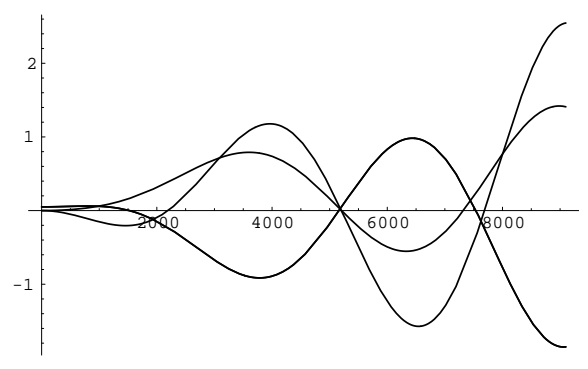

(a) For $t \in(0,9000 \mathrm{sec})$

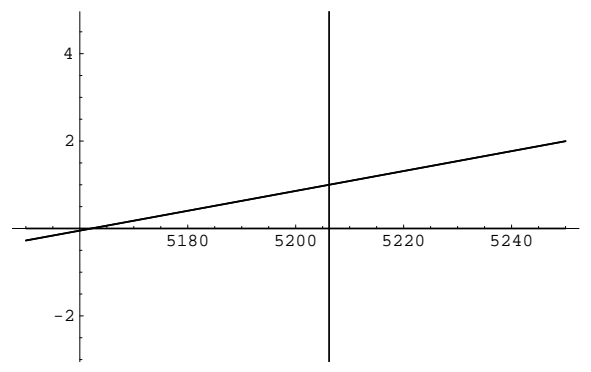

(b) For $t \in(5150,5250 \mathrm{sec})$

Figure 2: Determinant of $\Phi_{r r}, \Phi_{r v}, \Phi_{v r}$ and $\Phi_{v v}$ 


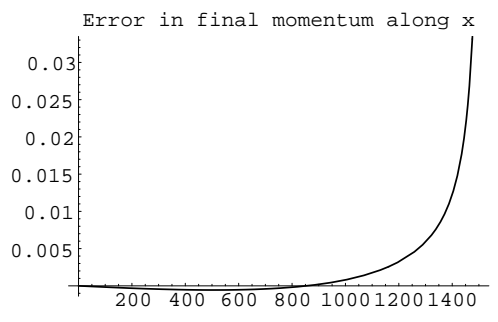

(a) Error in final momentum

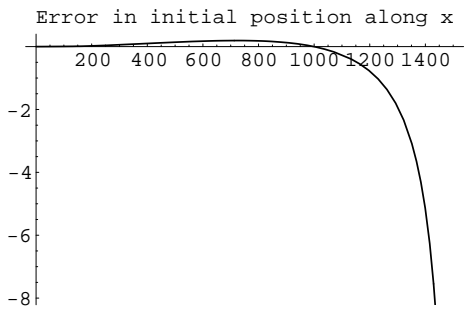

(c) Error in initial position

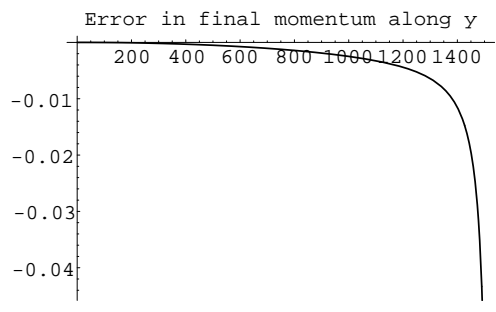

(b) Error in final momentum

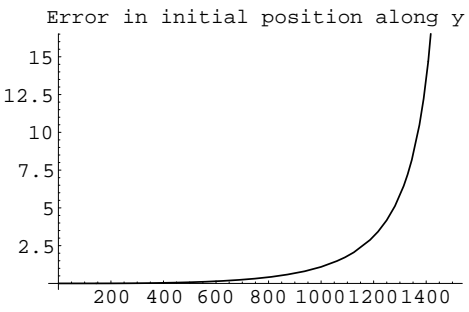

(d) Error in initial position

Figure 3: Order 2

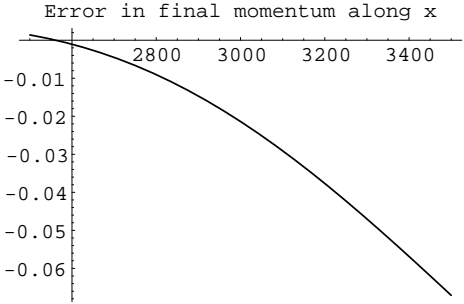

(a) Error in final momentum

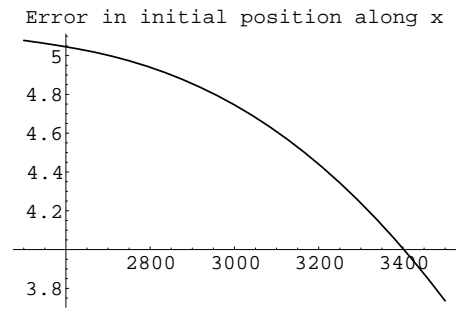

(c) Error in initial position

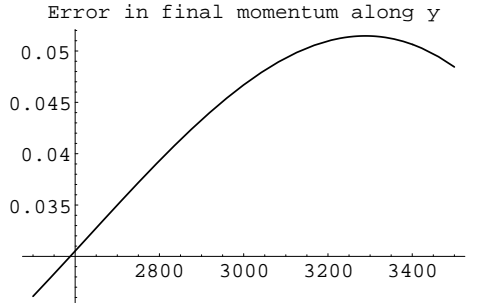

(b) Error in final momentum

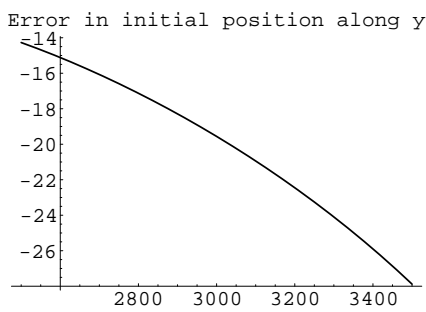

(d) Error in initial position

Figure 4: Order 2 


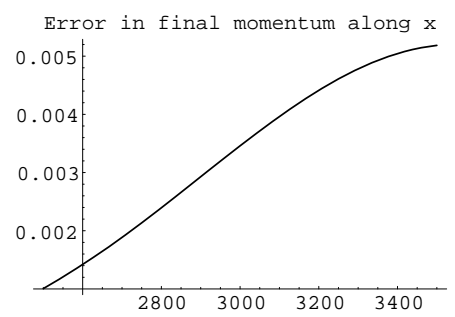

(a) Error in final momentum

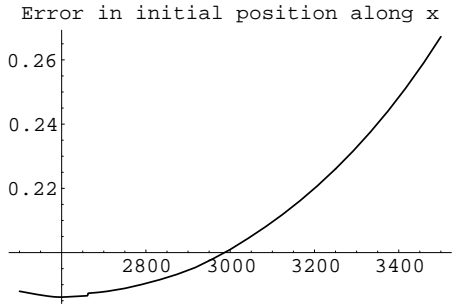

(c) Error in initial position

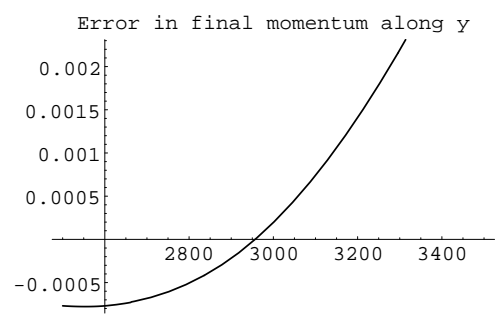

(b) Error in final momentum

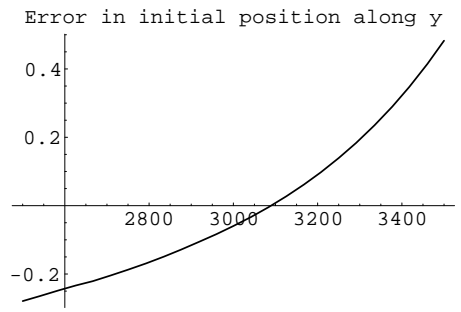

(d) Error in initial position

Figure 5: Order 3

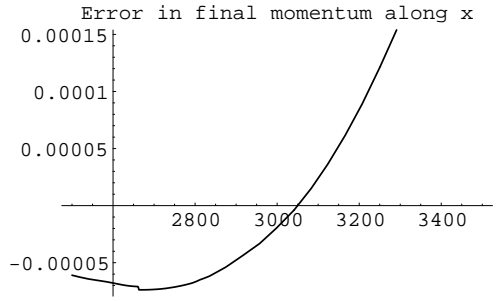

(a) Error in final momentum

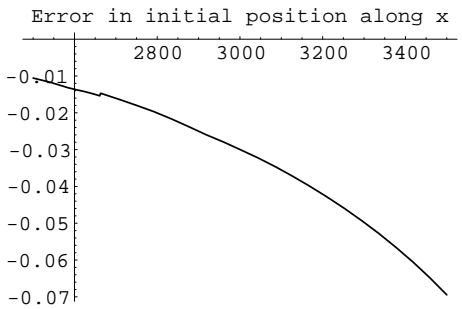

(c) Error in initial position

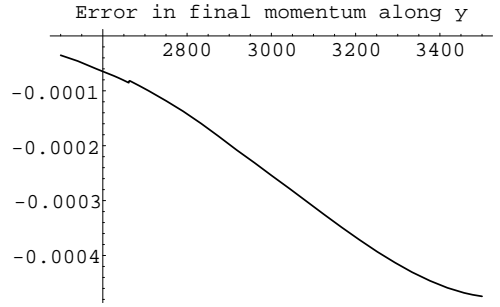

(b) Error in final momentum

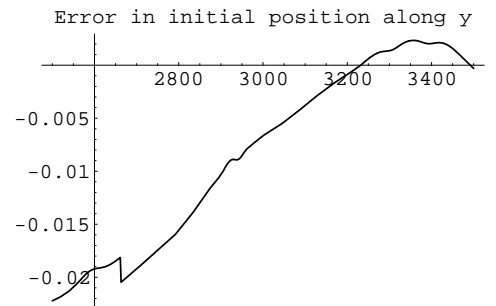

(d) Error in initial position

Figure 6: Order 4 